\title{
Beobachtungen von kleinen Planeten und Kometen.
}

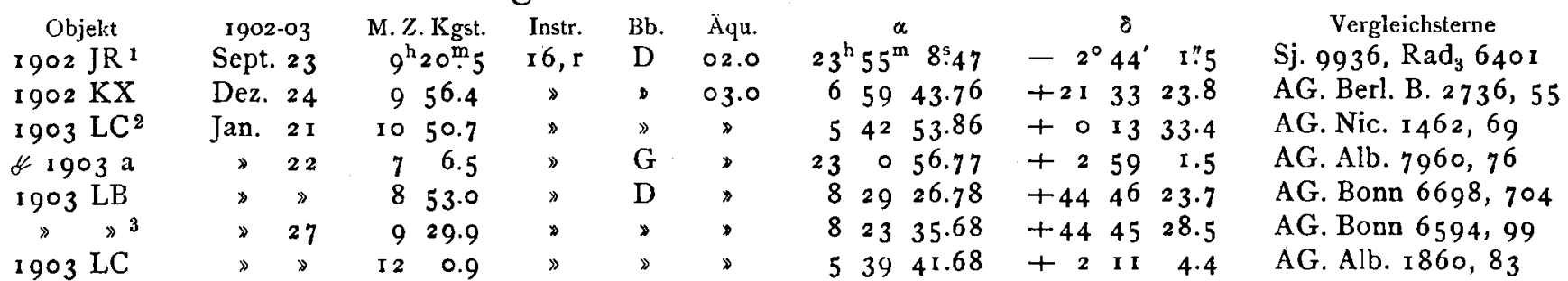

1 Unsicher. Planet schwach und am Rande der Platte. - ${ }^{2}$ Planetenstrich sehr lang, Mittelpunkt unsicher. - ${ }^{9}$ Schwach und schwierig.

Wegen der Kol. »Instr. « und $\gg$ Bb. * vgl. A. N. 3826 p. 183 .

Astrophysikalisches Observatorium Königstuhl, I903 Febr. 9.

M. Wolf.

\section{Uber eine nebelreiche Gegend in Lynx.}

Bei der photographischen Nachforschung nach dem interessanten Planeten (475) Occlo at der Grenze von Ursa major und Lynx fand ich mit dem Bruce-Teleskop die Nebelhäufigkeit ungemein groß. Es zeigen sich hier besonders viele kleine Nebel auf den Platten. Eine besonders dicht bestandene Gegend liegt um den Punkt $\left.\alpha=8^{\mathrm{h}} \mathbf{2}^{\mathrm{m}} \delta=+46{ }^{\circ} .^{*}\right)$ Die Mitte liegt also etwa zwischen den beiden Sternen der Bonner Durchmusterung: $\mathrm{BD} .+48 \div 1 \overline{3} 66\left(8 \mathrm{~m}_{5}\right)$ und $\mathrm{BD}$. $+48^{\circ} .1368\left(8^{\mathrm{mn}} 4\right)$. In einem Kreise mit 30 Bogenminuten Radius um diesen Punkt lassen sich mindestens 40 schwache kleine Nebel zählen.

Im allgemeinen sind sie viel schwächer und bedeutend weniger zahlreich als die Nebel um den Pol der Milchstraße $\alpha={ }_{12} 2^{\mathrm{h}} 53^{\mathrm{m}} \delta=+28{ }^{\circ} 5$, die ich vor zwei Jahren gefunden habe, und ein Zusammendrängen gegen ein Centrum scheint nicht $z u$ bestehen; aber immerhin ist der Reichtum der Gegend hervorragend.
Zwei der Nebel sind besonders bemerkenswert

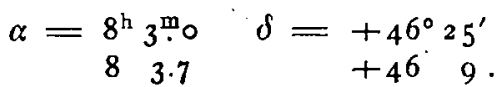

Der erste ist der bereits von W. Herschel beobachtete Nebel IV 55, NGC. 2537, der in lauter Sterne 20. Größe auflösbar sein soll. Er bietet auf der Platte einen ganz eigenartigen Anblick, wie ich ihn sonst noch nicht gesehen habe, etwa wie der Abdruck eines Vogelfußes. Er ist hell, ziemlich rund, hat etwa I' Durchmesser und verschiedene Verdichtungsstellen. Für einen großen Reflektor muß es ein sehr lohnendes Objekt sein.

Der andere ist meines Wissens noch unbekannt. Er ist etwa $3 \frac{1}{2}$ Bogenminuten lang, geradlinig, central verdichtet, sehr schmal und ziemlich hell. Er geht iber einen im nördlichen Teil gelegenen schwachen Stern im Positionswinkel $350^{\circ}$ und liegt etwa $I^{\prime}$ westlich von dem Stern BD. $+46^{\circ}$ I 37 I $\left(9^{\mathrm{m}} \cdot 3\right)$.

Astrophysikalisches Observatorium Königstuhl, 1903 Jan. 30.

Max Wolf.

*) Alle Koordinaten 1855.0 .

\section{Découverte d'une variable 2.1903 Draconis.}

En étudiant les photographies prises par M. S. Blajko, Mme. L. Ceraski a trouvé une variable nouvelle dont voici la position:

$$
\begin{aligned}
& 1855.0 \quad \alpha=9^{\mathrm{h}} 25^{\mathrm{m}} 4^{\mathrm{s}} .78 \quad \delta=+78^{\circ} 30^{\prime} \quad 6^{\prime \prime} 4 \\
& \text { I } 900.0 \quad 9315.09 \quad+78 \times 8 \times 4.3
\end{aligned}
$$

Cette étoile ne figure pas dans la BD., mais on la trouve dans l'AG. Cat. Kasan, sous le No. 1856 , où elle est taxée de 8.2 gr. ( 1870 oct. 30,31 ) et d'où sont tirées les coordonnées mentionnées.

Elle est suivie en AR. de quatre étoiles faibles dont les positions relatives sont:

Moscou, r 903 , le 24 janvier / 6 février.

$$
\begin{array}{lcccc}
\Delta \alpha \cos \delta+15^{\prime \prime} & 2 & 3 & 4 \\
\Delta \delta & +207^{\prime \prime} & +313^{\prime \prime} & +352^{\prime \prime} \\
\Delta \delta & +15^{\prime \prime} & +44^{\prime \prime} & +86^{\prime \prime} & +37^{\prime \prime}
\end{array}
$$

Nous avons douze plaques où la variable a les grandeurs suivantës :

$$
\begin{aligned}
& \text { 1 } 897 \text { mai } 4: \text { I } 3 \mathrm{mg} . \\
& 1898 \text { avril 1 } 1-15: \text { I } 2.5-13 \mathrm{mg} . \\
& \text { 1 } 899 \text { avril } 29-\text { mai } 5: 9.8 \mathrm{mg} \\
& \text { 1 } 900 \text { avril } 22-24: 9.3 \mathrm{mg} . \\
& \text { 1901 mai I } 5-16: 10.5 \mathrm{mg} .
\end{aligned}
$$

Prof. W. Ceraski.

In - F. Pidoux. Positions de quelques petites planètes. 119. - A. Abetti. Cometa 1903 a. 123. - A. A. Nijland. Beobachtung des Kometen 1903 a. 123. - G. Fayet. Eléments de la comète 1903 a. 123. - M. Ebell. Ephemeride des Kometen I902 b. 125. - P. Brück. Ephéméride de la comète 1903 a. 125. - F. Deichmïller. Beobachtung eines hellen Meteors. 125. - M. Wolf. Beobachtungen von kleinen Planeten und Kometen. 127. $-M$. Wolf. Über eine nebelreiche Gegend in Lynx. 127. - W. Ceraski. Decouverte d'une variable 2. L903 Draconis. 127. 